\title{
Evidence for the involvement of NGF in human sperm motility
}

\author{
Cui-Ge Shi ${ }^{1 *}$, Kai Lin ${ }^{2 *}$, Xiang-Bo Xu ${ }^{1}$, Shu-Cheng Zhang ${ }^{1}$, Ning Wang ${ }^{1}$, Ming Fan ${ }^{3 \#}$ \\ ${ }^{1}$ Department of Cell Biology, National Research Institute of Family Planning, Beijing, China \\ ${ }^{2}$ Clinic Laboratory Center, Air Force General Hospital, Beijing, China \\ ${ }^{3}$ Department of Brain Protection and Plasticity, Institute of Basic Medical Sciences, Beijing, China \\ Email: fanmingchina@126.com
}

Received 16 July 2012; revised 13 August 2012; accepted 20 August 2012

\begin{abstract}
Motility is an important physiological characteristic of a mature sperm. Nerve growth factor (NGF) is a key protein for the survival, maintenance and development of the central and peripheral nervous systems. It has been shown that NGF and its receptors TrkA and p75 are widely expressed in the testis, accessory reproductive organ, and the epididymal sperms. These observation have shifted the attention to the role of NGF on male reproductive physiology. In the present study, we found that NGF remarkably increased testicular coefficient in rats subjected to unpredictable chronic mild stress. Furthermore, we investigated the role of NGF on human sperm motility in vitro by CASA. The results showed that the parameters of sperm motility after NGF treatment had significantly increased, the means of VAP, VSL, VCL, BCF and LIN were significantly increased $32 \%$ than those of NGF absence, the means of MAD, STR, ALH and WOB had no notable difference. In addition, NGF promotes the sperm motility in a time- and dose-dependent manner. Taken together, our findings suggest that NGF plays a promoted role in sperm motility.
\end{abstract}

Keywords: NGF; Fertility; Testis; Sperm Motility

\section{INTRODUCTION}

In mammals, the family of the growth factor called neurotrophins (NTs) comprises four molecules, brain-derived neurotrophic factor (BDNF), neurotrophin 3, neurotrophins $4 / 5$ and nerve growth factor (NGF), which are involved in the nervous system maintenance and development [1]. However, the expression of NT receptors in non-neuronal tissues and the effects strongly suggests that NTs have a broader range of actions than originally

\footnotetext{
*Cui-Ge Shi and Kai Lin contributed equally to this work.

${ }^{*}$ Corresponding author.
}

supposed. More and more data suggest a role in the regulation of non-nervous tissues of these molecules $[2,3]$.

NGF bind to two kinds of receptors: The protein tyrosine kinase Trk receptors TrkA, act as specific high-affinity receptors. The low-affinity receptor $\mathrm{p} 75$, serves as a panneurotrophin receptor mediating pro-apoptotic or prosurvival cell programs [4]. NGF and its receptors had been reported to exist in the testis and NGF was expressed in Leydig cells, primary spermatocytes and pachytene spermatocytes [5]. TrkA only immunoreacted to elongate spermatids and $\mathrm{p} 75$ showed positive immunostaining in the Sertoli cells, Leydig cells, the pachytene spermatocytes and elongate spermatids [6,7]. NGF, TrkA and p75, have consistently been detected in developing and adult testicles of different mammalian species, and their expression has a hormonal regulation $[8,9]$. In addition, immunoreactions for NGF and its two receptors were also observed in columnar secretory epithelium lines of the seminal vesicles, prostate and coagulating gland. These results suggest that NGF is an important growth factor in male gonadal function $[10,11]$.

However, the key role of NGF on the human sperm motility remains unclear. In the present study, we attempt to elucidate the physiological role of NGF on human sperm motility in vitro.

\section{MATERIALS AND METHODS}

\subsection{Animals}

Adult male Sprague-Dawley (SD) rats with weights of $180-220 \mathrm{~g}$ were used. All animals were housed 5 per cage under standardized light/dark cycle condition (lights on at 7:00 AM, lights off at 7:00 PM) at a room temperature of $24^{\circ} \mathrm{C} \pm 1{ }^{\circ} \mathrm{C}$ and humidity of $60 \% \pm 10 \%$ with food and water ad libitum. This study was carried out in strict accordance with the recommendations in the Guide for the Care and Use of Laboratory Animals of the National Institutes of Health. The protocol was approved by the Committee on the Ethics of Animal Experiments of 
the National Research Institute of Family Planning. All surgery was performed under sodium pentobarbital anesthesia, and all efforts were made to minimize suffering.

The rats were randomly divided into four groups $(\mathrm{n}=$ 10): 1) control group; 2) unpredictable chronic mild stress (UCMS) group, in which rats were suffered different stress; 3) NGF group, in which rats were suffered differrent stress, and NGF were administrated intranasally at the dose of $50 \mathrm{mg} / \mathrm{kg} / \mathrm{d}$; 4) amitriptyline (AMI) group, in which rats were rats were suffered different stress, and AMI were administrated orally at the dose of $10 \mathrm{mg} / \mathrm{kg} / \mathrm{d}$.

\subsection{NGF Nasal Delivery}

For the rats, $1 \%$ mebumal sodium was dissolved in $0.9 \%$ $\mathrm{NaCl}$ was injected i.p. at the dosage of $40 \mathrm{mg} / \mathrm{kg}$ of body weight to induce anesthesia. Then, rats were laid on their back, with the head in upright position. NGF solution in PBS $(0.1 \mathrm{mg} / \mathrm{ml})$ were administered intranasally at the dose of $50 \mathrm{ug} / \mathrm{kg}, 10 \mathrm{ul}$ at a time, alternating the nostrils, with a lapse of 2 min between each administration, for a total of 10 times. During these procedures, the nostrils were always kept open.

\subsection{Unpredictable Chronic Mild Stress (UCMS)}

The stress protocol was designed to maximize unpredictability. It consisted of a variety of stressors applied randomly and at varying times of day for 14 days (Table 1). The following stressors were used: 1) $24 \mathrm{~h}$ of food deprivation; 2) $24 \mathrm{~h}$ of water deprivation; 3) $1-1.5 \mathrm{~h}$ of restraint; 4) 10 - 20 min inescapable foot shock; 5) $5 \mathrm{~min}$ warm swim $\left(42^{\circ} \mathrm{C}\right)$; 6) 5 min cold $\operatorname{swim}\left(16^{\circ} \mathrm{C}\right)$; 7) $1 \mathrm{~min}$ tail clipping. All stressors were applied to animals in a separate procedure room. Control rats were handled daily in the housing room for 14 days.

\subsection{Subjects}

20 normal male subjects aging 25 - 40-year-old with fertility were investigated between January and June 2011 at Air Force General Hospital. The sperm samples have motility higher than $60 \%$ and count over 20 millions $/ \mathrm{ml}$. The individual in this manuscript has given written informed consent to publish these case details.

\subsection{Preparation of Semen Samples}

Fresh semen samples were collected by masturbation into sterile plastic containers after 7 days of sexual abstinence and were allowed to liquefy for 30 minutes at room temperature [12]. Semen analysis was performed according to WHO protocols. Samples were processed according to the routine wash and swim-up procedure in HEPES-HTF (Santa Ana, CA, USA) medium supplemented with $10 \%$ serum substitute supplement (Santa
Table 1. The procedure of UCMS.

\begin{tabular}{clc}
\hline Day & Type of stressor & Time \\
\hline 1 & 60 min restraint & $\mathrm{am}$ \\
2 & $\begin{array}{l}20 \text { min inescapable foot shock }(1.0 \mathrm{~mA}, 15 \mathrm{~s}, \\
60 \mathrm{~s} \text { off })\end{array}$ & $\mathrm{pm}$ \\
3 & 24 -h food deprivation & \\
4 & 5 min warm swim $\left(42^{\circ} \mathrm{C}\right)$ & $\mathrm{am}$ \\
5 & 24 -h food deprivation & \\
6 & 5 min cold swim $\left(16^{\circ} \mathrm{C}\right)$ & $\mathrm{pm}$ \\
7 & $\begin{array}{l}1 \text { min tail clipping }(\mathrm{a} \text { clothespin placed at } 1 \mathrm{~cm} \\
\text { from the base of tail) }\end{array}$ & $\mathrm{am}$ \\
8 & $24-\mathrm{h}$ food deprivation & \\
9 & $\begin{array}{l}10 \text { min inescapable foot shock }(1.0 \mathrm{~mA}, 15 \mathrm{~s}, \\
60 \mathrm{~s} \text { off })\end{array}$ & $\mathrm{pm}$ \\
10 & 5 min warm swim $\left(42^{\circ} \mathrm{C}\right)$ & $\mathrm{am}$ \\
11 & 90 min restraint & $\mathrm{pm}$ \\
12 & 1 min tail clipping $(\mathrm{a}$ clothespin placed at $1 \mathrm{~cm}$ & $\mathrm{am}$ \\
& from the base of tail $)$ & $\mathrm{pm}$ \\
13 & 5 min cold swim $\left(16^{\circ} \mathrm{C}\right)$ & \\
14 & $24-\mathrm{h}$ food deprivation &
\end{tabular}

Ana, CA, USA).

\subsection{Determination of Sperm Motility Parameters}

$10 \mathrm{ml}$ of sperm suspension was placed on a Makler chamber and sperm motility parameters were analyzed by Computer-aided sperm analysis (CASA), following incubation for 1 hour at 37 in air. The semen analyzer used was the Hamilton Thorne Research semen analyzer (IVOS, Version 10.8x. Hamilton Thorne, Beverly, USA). The standard parameter settings employed for analysis were as follows: frame acquired, 30; frame rate, $60 \mathrm{~Hz}$; minimum contrast, 80 ; minimum cell size, 3 ; static head size limits, $1.00 \sim 2.90$; static head intensity limits, 0.60 $\sim 1.40$; static elongation limits, $0-80$ and temperature, $37^{\circ} \mathrm{C}$. At least 10 fields were examined.

The post swim-up sperm suspensions were used to assess the sperm motility, as it was proposed that the post swim-up motility is a better predictor of fertilizing ability of spermatozoa than fresh semen. The following motility parameters were measured: concentration (CON, Million $/ \mathrm{mL}$ ), rate of motility (\%MOT) and rate of rapid progression (\%PMOT). For those spermatozoa that exhibit an average path velocity (VAP) greater than $25 \mathrm{~mm} / \mathrm{s}$, the following parameters were determined: average path velocity (VAP, $\mathrm{mm} / \mathrm{s}$ ), straight line velocity (VSL, $\mathrm{mm} / \mathrm{s}$ ), curvilinear velocity $(\mathrm{VCL}, \mathrm{mm} / \mathrm{s})$, amplitude of lateral head displacement (ALH, mm), beat-cross frequency (BCF, Hz), Straightness (STR, \%); VSL/VAP and Linearity (LIN, \%). 
A more specified measure is motility grade, where the motility of sperm is divided into four different grades:

Grade a: Sperm with progressive motility. These are the strongest and swim fast in a straight line. Grade b: (non-linear motility): These also move forward but tend to travel in a curved or crooked motion. Grade c: These have non-progressive motility because they do not move forward despite the fact that they move their tails. Grade d: These are immotile and fail to move at all. Sometimes also denoted motility IV.

\subsection{Statistical Analyses}

For statistical analyses, a standard software package (SPSS for Windows 10.1) was used. All data were given as means $\pm \mathrm{SD}$. Differences between groups were compared by using a one-way analysis of variance (ANOVA). $P$ values $<0.05$ were considered significant.

\section{RESULTS}

\subsection{Effects of NGF on Testicular Organ Coefficient}

Intranasal NGF remarkably increased testicular coefficient in rats subjected to UCMS. The testicular coefficient of the rats were $1.1 \pm 0.1$ in control group, which were much higher than those subjected to UCMS $(0.8 \pm$ 0.2 ). During the 2-week treatment period of NGF, the testicular coefficient increased to $1.2 \pm 0.14$. As a positive control, AMI $(10 \mathrm{mg} / \mathrm{kg})$ increased the testicular coefficient to $1.3 \pm 0.1$ (Figure 1).

\subsection{Dose-Dependent Effect of NGF on Sperm Motility}

Percoll-washed spermatozoa that exhibited more than $70 \%$ motility were used for the experiments. As compared with the control, a significant dose-dependent improvement in sperm motility was noticed in the experimental fractions supplemented with $0.1,1$ and $10 \mu \mathrm{M}$ concentrations of NGF for $30 \mathrm{~min}$ (Figure 2). The sperm number of grade $\mathrm{A}$ was increased and the sperm number of grade $\mathrm{C}$ and $\mathrm{D}$ was decreased (Figure 3 ). In particular, the means of VAP, VSL, VCL, BCF and LIN were significantly increased more than $32 \%$. The means of MAD, STR, ALH and WOB had no notable difference (Figure 4).

\subsection{Time-Dependent Effect of NGF on Sperm Motility}

The mean percentage of motile sperm were assessed by CASA after $10 \mu \mathrm{M}$ NGF for $2,10,20,30,40,50$ and 60 min incubation, respectively (Figure 5). As compared with the control, the means of VAP, VSL, VCL, BCF and LIN were significantly increased and the means of

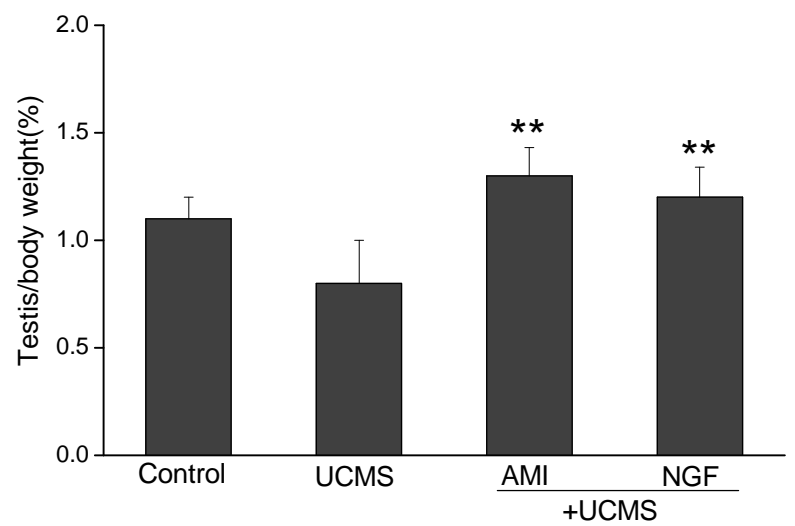

Figure 1. Effects of intranasal NGF $(50 \mu \mathrm{g} / \mathrm{kg})$ on the testicular coefficient in rats after UCMS. As a positive control, AMI (10 $\mathrm{mg} / \mathrm{kg}$, po.) was given for 14 days. NGF remarkably increased testicular coefficient in rats subjected to UCMS. Vertical bars represent means $\pm \mathrm{SE} . * \mathrm{P}<0.05, * * \mathrm{P}<0.01$ vs control. $\# \mathrm{P}<$ $0.05, \# \mathrm{P}<0.01$ vs UCMS. $\mathrm{n}=8$.
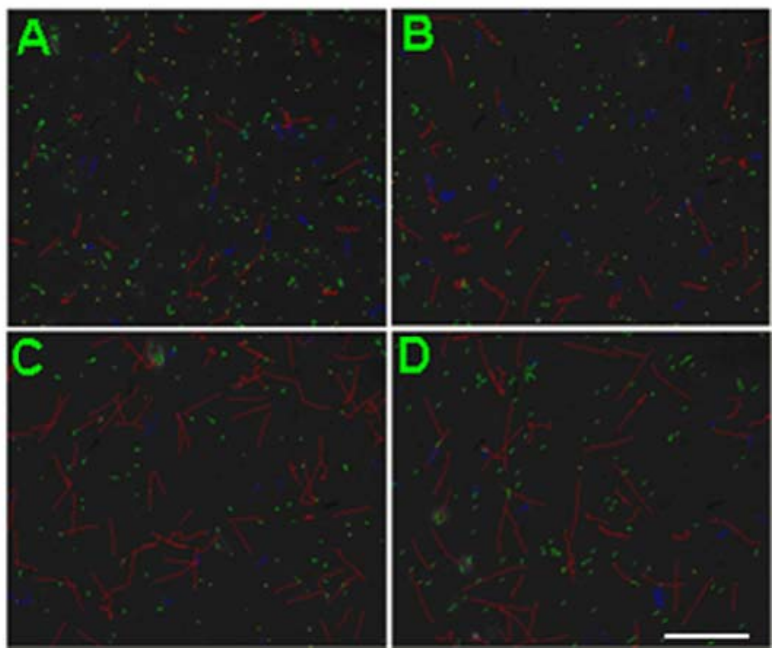

Figure 2. Representative CASA tracks of sperm after half an ne hour of NGF treatment. (A) Control sperm, buffer only; (B) Sperm treated with $0.1 \mu \mathrm{M}$ NGF; (C) Sperm treated with $1 \mu \mathrm{M}$ NGF; (D) Sperm treated with $10 \mu \mathrm{M}$ NGF. The bar represents $50 \mu \mathrm{m}$.

MAD, STR, ALH and WOB had no notable difference (Figure 6).

\section{DISCUSSION}

These studies evaluated that NGF intranasal delivery could remarkably increased testicular coefficient in rats subjected to UCMS. In addition, we revealed an evidence that demonstrate a direct role of NGF on human sperm motility. NGF treatment could maintain and enhance sperm motility in time- and dose-dependent manner.

UCMS has been reported to be a risk factor for infertility. Studies have shown that exposure to UCMS causes testis weight declining, which is associated with directly 


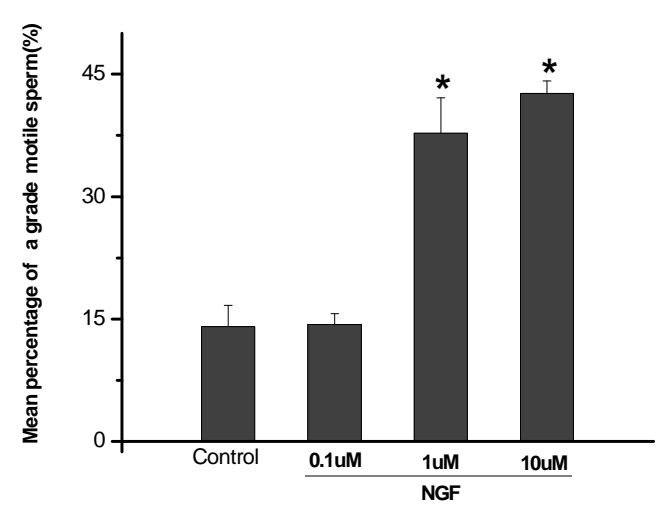

(a)

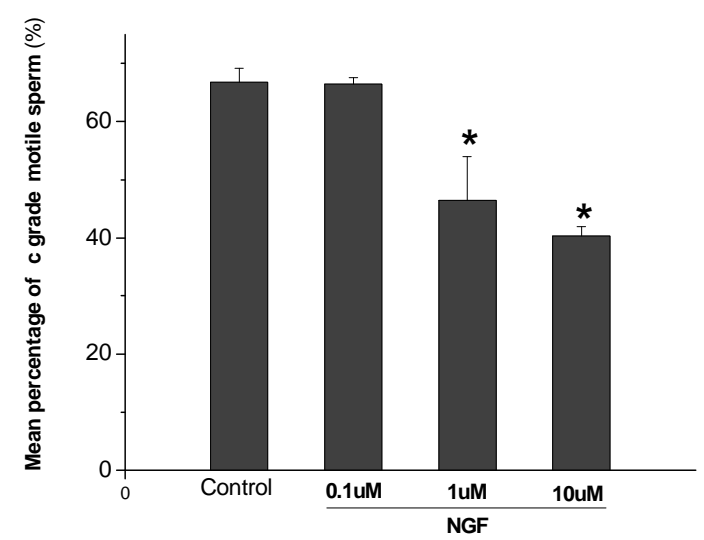

(c)

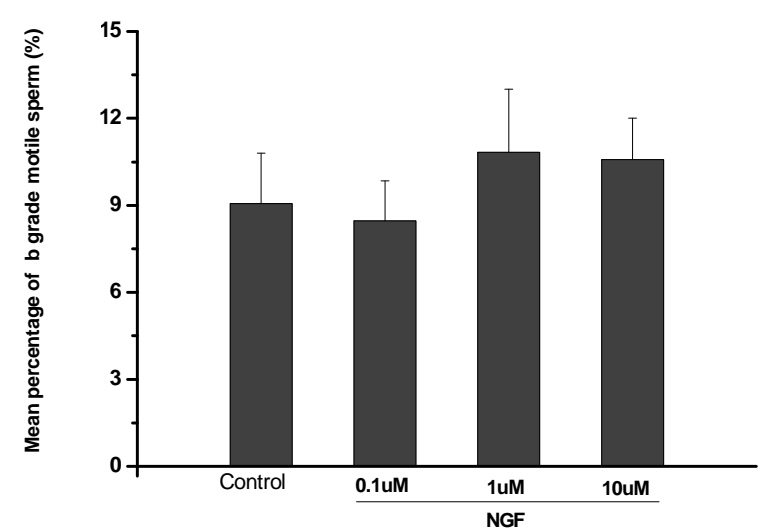

(b)

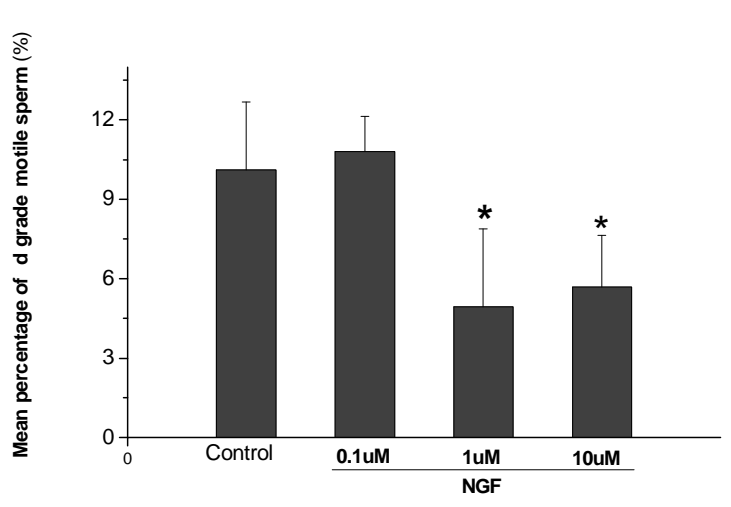

(d)

Figure 3. Effects of NGF on the mean percentage of motile sperm of (a)-(d) grades in dose-dependent manner. The mean percentage of motile sperm of (a) grades was significantly increased and the mean percentage of motile sperm of (c) and (d) grade was decreased compared with control. Vertical bars represent means $\pm \mathrm{SE}$. $* \mathrm{P}<0.05, * * \mathrm{P}<0.01$ vs control. $\mathrm{n}=10$.

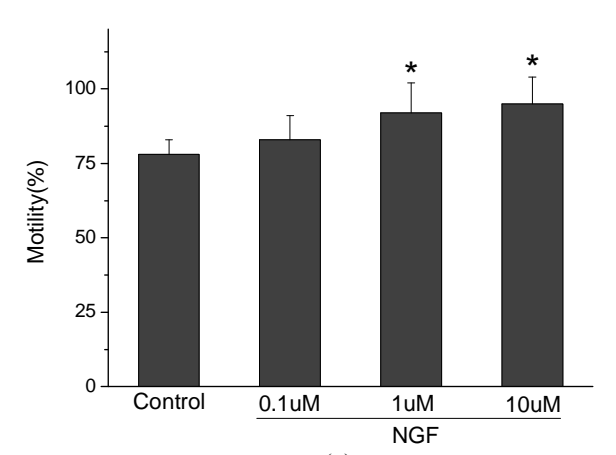

(a)

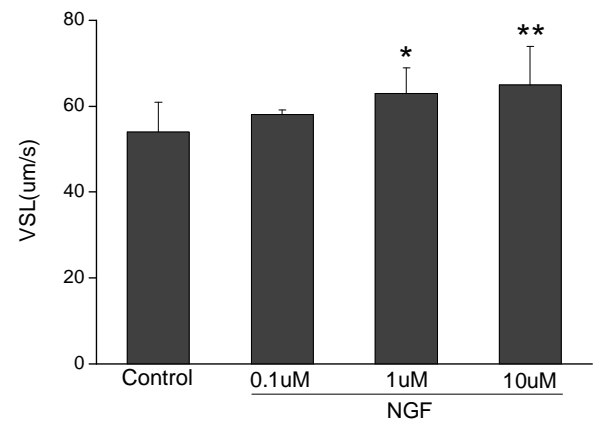

(c)

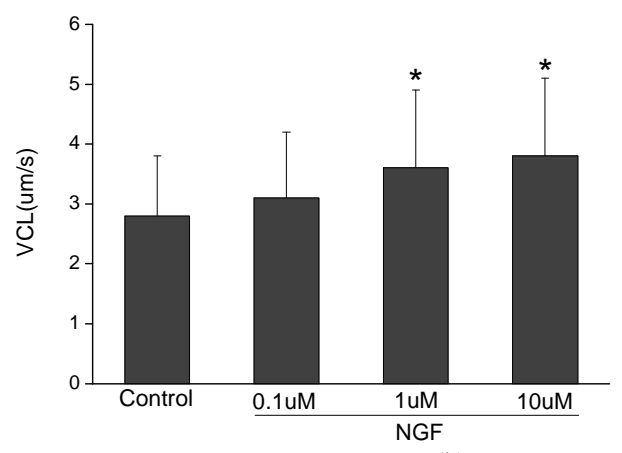

(b)

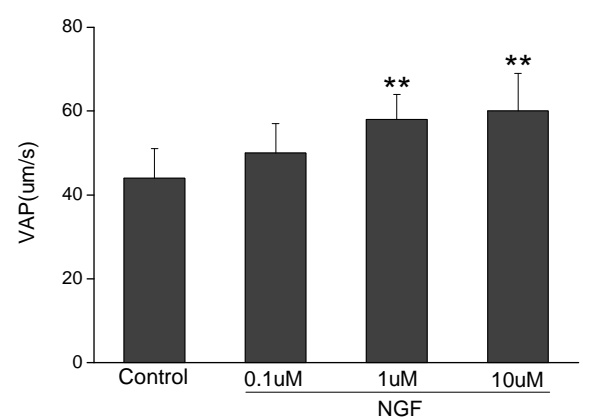

(d) 


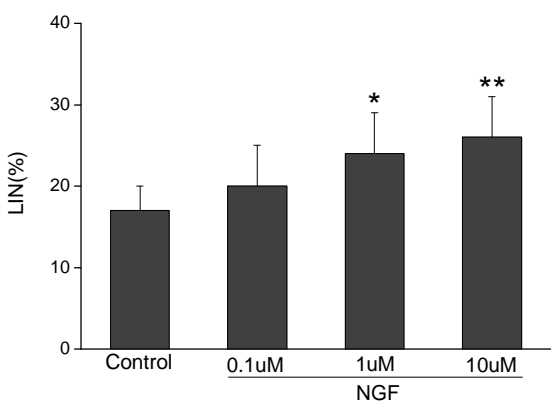

(e)

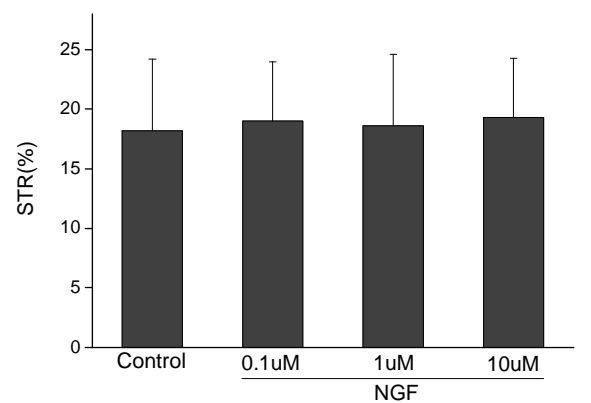

(g)

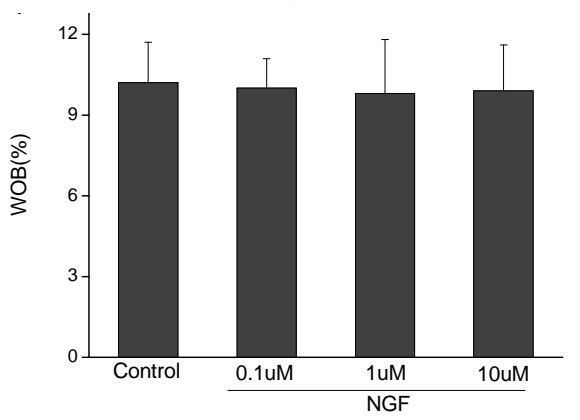

(i)

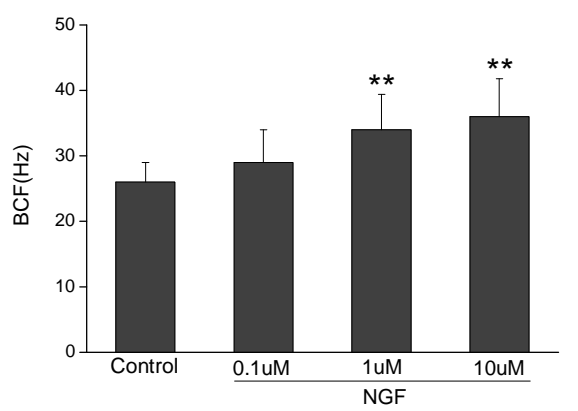

(f)

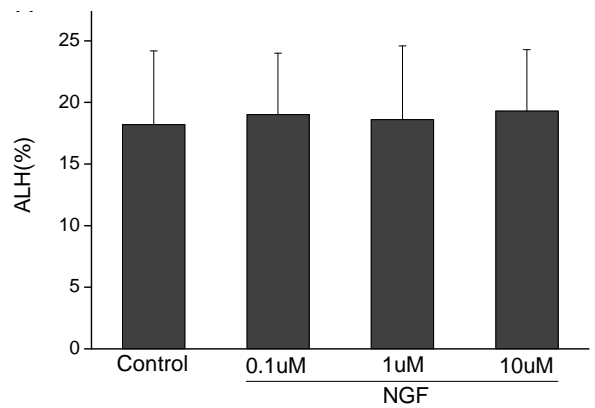

(h)

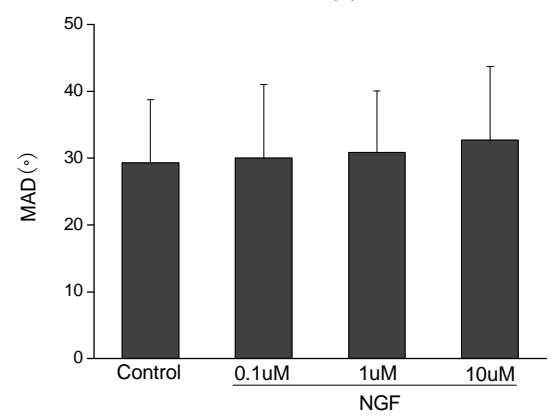

(j)

Figure 4. Sperm were treated with increasing concentration of NGF and assessed using CASA. The percentage of motile sperm and sperm motility parameters include VCL, VSL, VAP, LIN and $\mathrm{BCF}$ were significantly increased by NGF treatment in a dose-dependent manner. Vertical bars represent means $\pm \mathrm{SE} . * \mathrm{P}<0.05, * * \mathrm{P}<0.01$ vs control. $\mathrm{n}=20$.

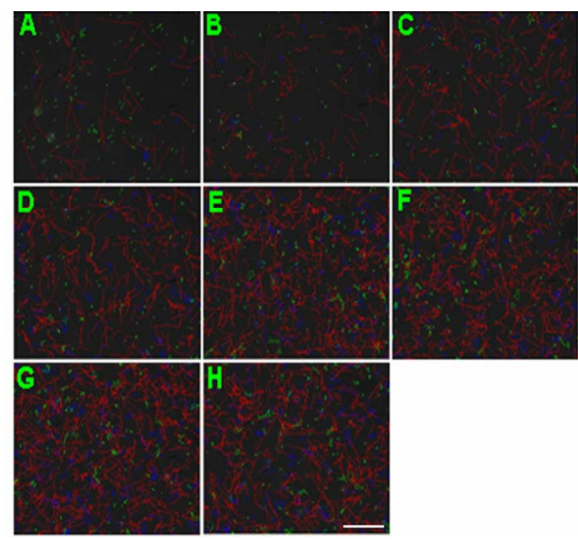

Figure 5. Representative CASA tracks of sperm after $10 \mu \mathrm{M}$ NGF treatment over time. (A) control; (B) $2 \mathrm{~min}$; (C) $10 \mathrm{~min}$; (D) 20 min, (E) $30 \mathrm{~min}$; (F) $40 \mathrm{~min}$; (G) $50 \mathrm{~min}$; (H) $60 \mathrm{~min}$. The bar represents $50 \mu \mathrm{m}$. damage the testis. In our study, NGF could increased testicular coefficient, this concept may be involved in the neuroendocrine regulation of NGF. The involvement of NGF in neuroendocrine regulation is supported by several lines of evidence. NGF content and expression were found in the pituitary gland and other endocrine organs during dysendocrine states (thyroidectomized, adrenalectomized and gonadectomized male rats). NGF intranasal delivery could increased the content in pituitary gland, which could regulate gonadectomized incretion [12].

Taken together, our data suggest that NGF pathway might be involved in sperm motility. This concept may be supported by NGF and its receptors expression. A positive immunoreactivity for NGF has been reported in the adult mouse testis. This immunoreactivity appeared to be localized to the cells of the germ line. It was positive from primary spermatocytes to mature sperm. The 

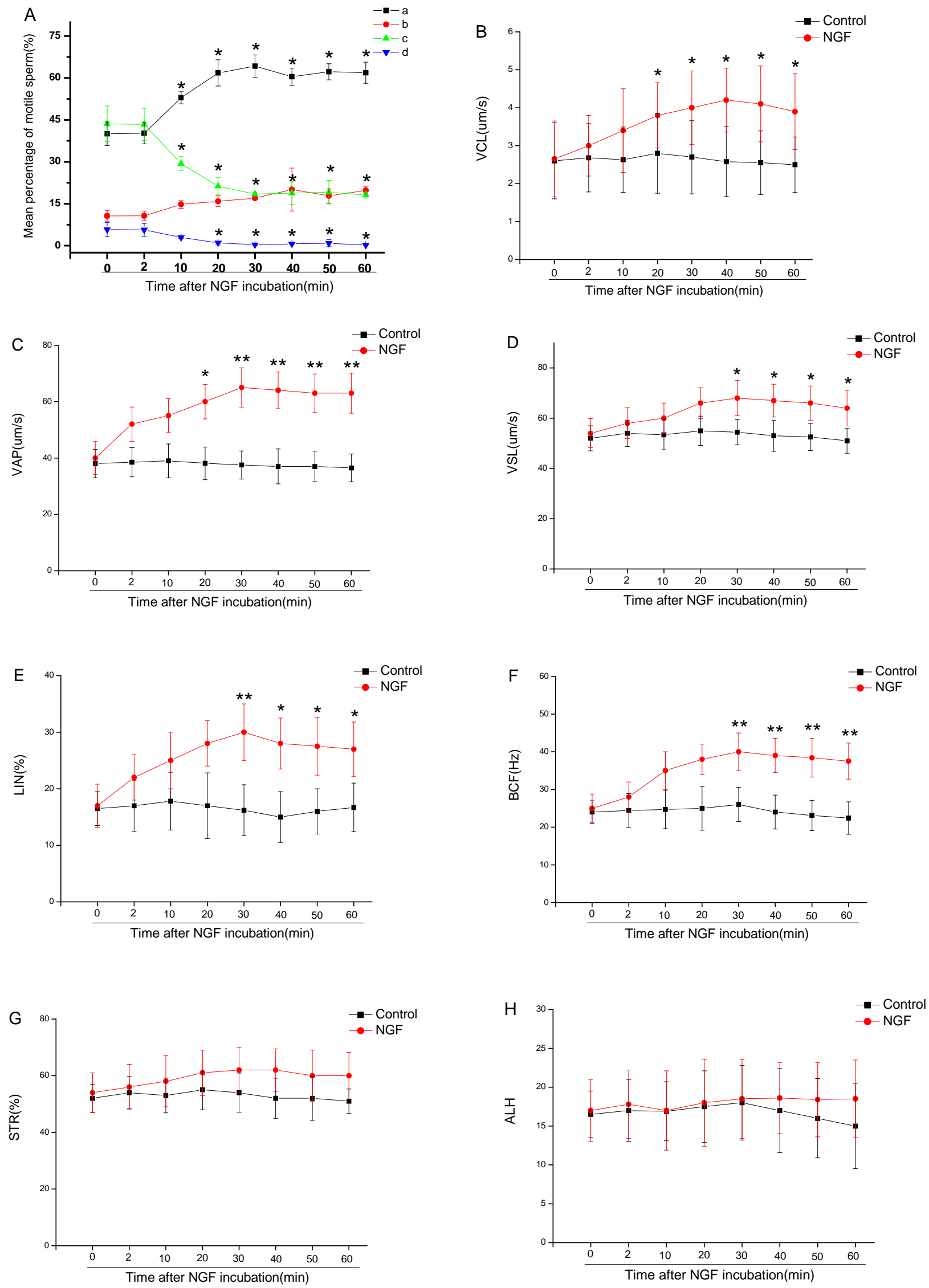

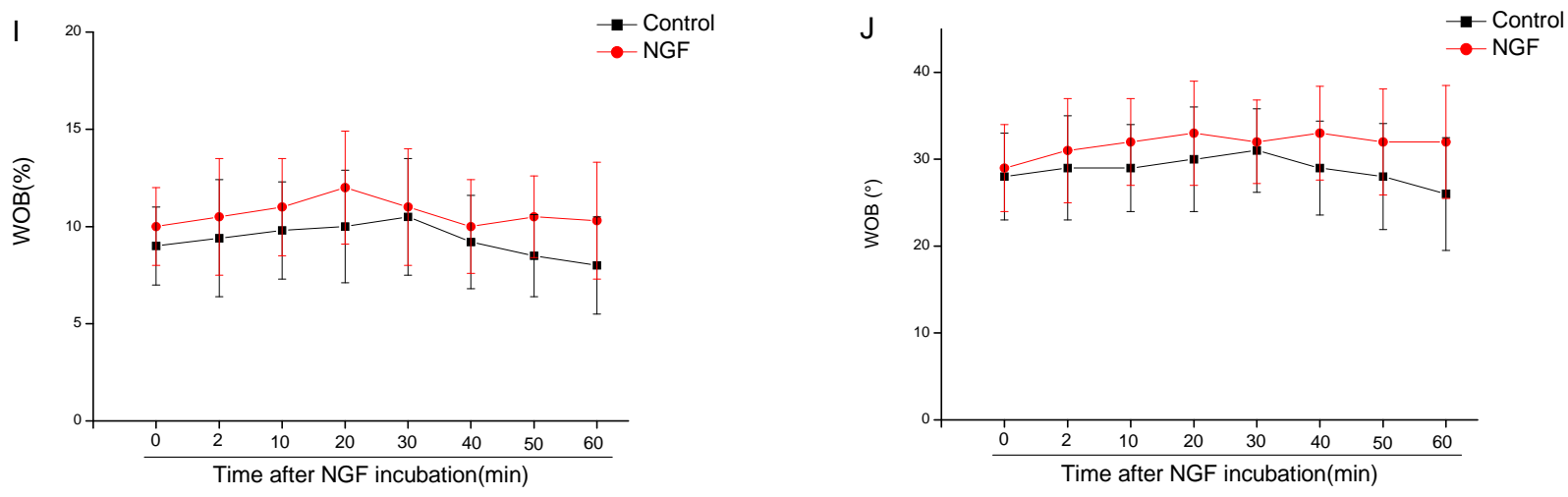

Figure 6. Sperm were treated with increasing concentration of $10 \mu \mathrm{M}$ NGF and assessed using CASA over time. The mean percentage of motile sperm of $\mathrm{a}$ and $\mathrm{b}$ grades was significantly increased and the mean percentage of motile sperm of $\mathrm{c}$ and $\mathrm{d}$ grade was decreased. The majority of sperm motility parameters include VCL, VSL, VAP, LIN and BCF were enhanced by NGF treatment, compared with control. Vertical bars represent means $\pm \mathrm{SE}$. $* \mathrm{P}<0.05, * * \mathrm{P}<0.01$ vs control. $\mathrm{n}=20$.

presence of NGF mRNA and protein in adult rodent testis has also been demonstrated. NGF mRNA was detected in spermatocytes and early spermatids of adult mouse and rat testis $[12,13]$. The presence of NGF-like substance in a testicular extract was also confirmed by a bioassay. An analysis of the stage-specific expression of NGF during the cycle of the seminiferous epithelium in the rat revealed NGF mRNA and protein at all stages of the cycle. A quantitative determination by immunoassay of NGF in human testis revealed the presence of $5.44 \mathrm{ng}$ of NGF per $g$ wet weight $[14,15]$.

NGF remains associated with spermatozoa throughout their maturation in the epididymis, and further peptides may be absorbed to the sperm surface from sources in the epididymal epithelium. The presence of NGF in spermatozoa and in prostatic secretions raises important questions concerning the role of this protein in sperm motility, particularly since NGF is a known chemoattractant for motile leucocytes. The possible relationship between NGF and the "forward motility protein" also needs to be addressed carefully [16].

In summary, NGF have critical role in sperm motility. These results may promote the application of NGF in assisted reproductive technology in clinic.

\section{FUNDING SOURCES}

The work was supported by the Central Public-interest Scientific Institution Basal Research Fund (2010GJSS- JKA03) and the National Key Grant of Basic Research Project (2006CB504100).

\section{REFERENCES}

[1] Pardon, M.C. (2010) Role of neurotrophic factors in behavioral processes: Implications for the treatment of psychiatric and neurodegenerative disorders. Vitamins \& Hormones, 82, 185-200.

doi:10.1016/S0083-6729(10)82010-2
[2] Spinnler, K., Köhn, F.M., Schwarzer, U. and Mayerhofer, A. (2010) Glial cell line-derived neurotrophic factor is constitutively produced by human testicular peritubular cells and may contribute to the spermatogonial stem cell niche in man. Human Reproduction, 25, 2181-2187. doi:10.1093/humrep/deq170

[3] Jin, W., Tanaka, A., Watanabe, G., Matsuda, H. and Taya, K. (2010) Effect of NGF on the motility and acrosome reaction of golden hamster spermatozoa in vitro. Journal of Reproduction and Development, 56, 437-443. doi:10.1262/jrd.09-219N

[4] Manca, A., Capsoni, S., Di Luzio, A., et al. (2012) Nerve growth factor regulates axial rotation during early stages of chick embryo development. Proceedings of the $\mathrm{Na}$ tional Academy of Sciences of USA, 7109, 2009-2014. doi:10.1073/pnas.1121138109

[5] Gnessi, L., Fabbri, A. and Spera, G. (1997) Gonadal peptides as mediators of development and functional control of the testis: An integrated system with hormones and local environment. Endocrine Reviews, 18, 541-609. doi:10.1210/er.18.4.541

[6] Artico, M., Bronzetti, E., Saso, L., et al. (2007). Immunohistochemical profile of some neuro-transmitters and neurotrophins in the seminiferous tubules of rats treated by lonidamine. European Journal of Histochemistry, 51, 19-24.

[7] Perrard, M.H., Vigier, M., Damestoy, A., et al. (2007) BetaNerve growth factor participates in an auto/paracrine pathway of regulation of the meiotic differentiation of rat spermatocytes. Journal of Cellular Physiology, 210, 51-62. doi: $10.1002 /$ jep. 20805

[8] Jin, C.W., Arai, K.Y., Shimizu, K., et al. (2006) Cellular localization of NGF and its receptors trkA and p75LNGFR in male reproductive organs of the Japanese monkey, Macaca fuscata fuscata. Endocrine, 29, 155-160. doi:10.1385/ENDO:29:1:155

[9] Levanti, M.B., Germanà, A., De Carlos, F., et al. (2006) Effects of increased nerve growth factor plasma levels on the expression of TrkA and p75 in rat testicles. Journal of Anatomy, 208, 373-379.

doi:10.1111/j.1469-7580.2006.00528.x 
[10] Spinnler, K., Fröhlich, T., Arnold, G.J., et al. (2011) Human tryptase cleaves pro-nerve growth factor (pro-NGF): Hints of local, mast cell-dependent regulation of NGF/ pro-NGF action. The Journal of Biological Chemistry, 286, 31707-31713. doi:10.1074/jbc.M111.233486

[11] Hou, Y., Bao, X.Q., Wei, H.L., Luo, Y. and Liu, G.T. (2011) Long-term deprivation of gonadal hormone accelerates brain aging in mice. Research on Neurology, 33, 43-49. doi:10.1179/016164110X12700393823499

[12] Calzà, L., Giardino, L, Aloe, L. (1997) NGF content and expression in the rat pituitary gland and regulation by thyroid hormone. Molecular Brain Research, 51, 60-68. doi:10.1016/S0169-328X(97)00213-1

[13] Koeva, Y.A. (2002) Immunolocalization of neurotrophic factors and their receptors in the Leydig cells of rat during postnatal development. Folia Medica (Plovdiv), 44,

\section{Abbreviations}

NGF: Nerve growth factor;

UCMS: Unpredictable chronic mild stress;

VAP: Average path velocity;

VSL: Straight line velocity;

VCL: Curvilinear velocity;
27-31.

[14] Parvinen, M., Pelto-Huikko, M., Söder, O.S., et al. (1992) Expression of beta-nerve growth factor and its receptor in rat seminiferous epithelium: Specific function at the onset of meiosis. The Journal of Cell Biology, 117, 629-641. doi:10.1083/jcb.117.3.629

[15] Kim, S.T., Park, N.C., Yi, L.S. and Gye, M.C. (2006). Expression of p57kip2 in germ cells and Leydig cells in human testis. Archives of Andrology, 52, 463-469. doi:10.1080/01485010600822903

[16] Grabham, P.W., Foley, M., Umeojiako, A. and Goldberg, D.J. (2000) Nerve growth factor stimulates coupling of beta1 integrin to distinct transport mechanisms in the filopodia of growth cones. Journal of Cell Science, 113, 30031302 .

ALH: Amplitude of lateral head displacement;

BCF: Beat-cross frequency;

STR: Straightness;

LIN: Linearity;

CASA: Computer assisted sperm analysis. 